\title{
Erkrankungen der Aorta: von der Aortenklappe bis zur Aortenbifurkation
}

Autoren

Christine Quast ${ }^{1}$, Sebastian Zimmer ${ }^{2}$, Matti Adam³ , Stephan Baldus ${ }^{3}$, Georg Nickenig ${ }^{2}$, Malte Kelm¹, 4

Institute

1 Klinik für Kardiologie, Pneumologie und Angiologie, Universitätsklinikum Düsseldorf, Heinrich-HeineUniversität Düsseldorf

2 Herzzentrum Bonn, Medizinische Klinik und Poliklinik II, Universitätsklinikum Bonn

3 Klinik III für Innere Medizin - Allgemeine und interventionelle Kardiologie, Elektrophysiologie, Angiologie, Pneumologie und internistische Intensivmedizin, Uniklinik Köln

4 CARID, Cardiovascular Research Institute Düsseldorf

Bibliografie

DOI https://doi.org/10.1055/a-0663-6474

Dtsch Med Wochenschr 2019; 144: 734-738

(c) Georg Thieme Verlag KG, Stuttgart · New York

ISSN 0012-0472
WAS IST NEU?

Stand der Dinge Sowohl im klinischen Bereich als auch in der Forschung etabliert sich zunehmend das Konzept, die Aorta als Gesamtorgan zu betrachten, das sich von der Aortenklappe bis zur Bifurkation erstreckt.

Aortenklappenstenose Aktuell laufen erste randomisierte Studien zur Ausweitung der Indikation zum interventionellen Aortenklappenersatz (TAVI, TAVR) hin zu einem Patientenkollektiv mit niedrigem OP-Risiko. Des Weiteren hat die interventionelle Versorgung der „Low flow, low gradient“-Aortenklappenstenose eine Aufwertung im Empfehlungsgrad erhalten und die interventionelle Valve-in-valve-Prozedur Eingang in den klinischen Alltag bei der individualisierten Patientenversorgung gefunden.

Aortenklappeninsuffizienz Kathetergestützt implantierte Klappenprothesen, die dediziert zur Behandlung der isolierten Aortenklappeninsuffizienz entwickelt werden, befinden sich für dieses Krankheitsbild in der klinischen Prüfung.

Erkrankungen der Aorta ascendens und descendens Während das Screening von Aortenaneurysmen zur ambulanten Routine wird, sind Studien zum risikostratifizierten Followup bei zunehmend endovaskulär versorgten Patienten notwendig, um eine bedarfsgerechte Nachsorge zu ermöglichen.

\section{Stand der Dinge}

Sowohl im klinischen als auch im Forschungsbereich etabliert sich zunehmend das Konzept, die Aorta als komplettes Organ von der Aortenklappe bis zur Bifurkation zu betrachten und zu behandeln. Innerhalb der letzten Jahre wurde damit begonnen, die zugrunde liegenden Pathomechanismen aufzuklären, um frühe Indikatoren aortaler Erkrankungen ausfindig zu machen und eine Therapie in früheren Erkrankungsstadien einzuleiten. Obwohl jeder Bereich der Aorta eine spezifische embryogenetische, histologische und funktionelle Charakteristik aufweist, ist die Aorta insgesamt ähnlichen mechanischen Kräften ausgesetzt. Biologische, chemische und mechanische Stressoren führen zur Dysfunktion der aortalen endothelialen Auskleidung, Rekrutierung und Aktivierung von Immunzellen und Modifikation des interstitiellen zellulären Metabolismus mit krankhaftem Matrixremodeling und verfrühter Kalzifizierung. Dies sind kritische Voraussetzungen für eine Schädigung an Aortenwand und Aortenklappe.
Während die Aortenklappenstenose eine umschriebene Erkrankungsentität darstellt, umfassen die weiter distal gelegenen Erkrankungen der Aorta ( $\bullet$ Abb. 1) ein heterogenes Erkrankungsbild bestehend aus

- der Aortenektasie der Aorta ascendens,

- dem thorakalen und/oder abdominellen Aortenaneurysma,

- der Aortendissektion,

- dem intramuralen Hämatom (IMH),

- dem penetrierenden Aortenulkus (PAU) und

- der Aortenruptur.

\section{Aortenklappenstenose}

\section{Aktuelle Entwicklungen bei der Versorgung der Aortenklappenstenose}

Im Bereich der interventionellen Versorgung von Aortenklappenstenosen hat der interventionelle Aortenklappenersatz bei symptomatischer „Low flow, low gradient“Aortenklappenstenose in der ESC-Leitlinie von 2017 eine Aufwertung erfahren (von Ilb C auf Ila C). Das Vorhan- 


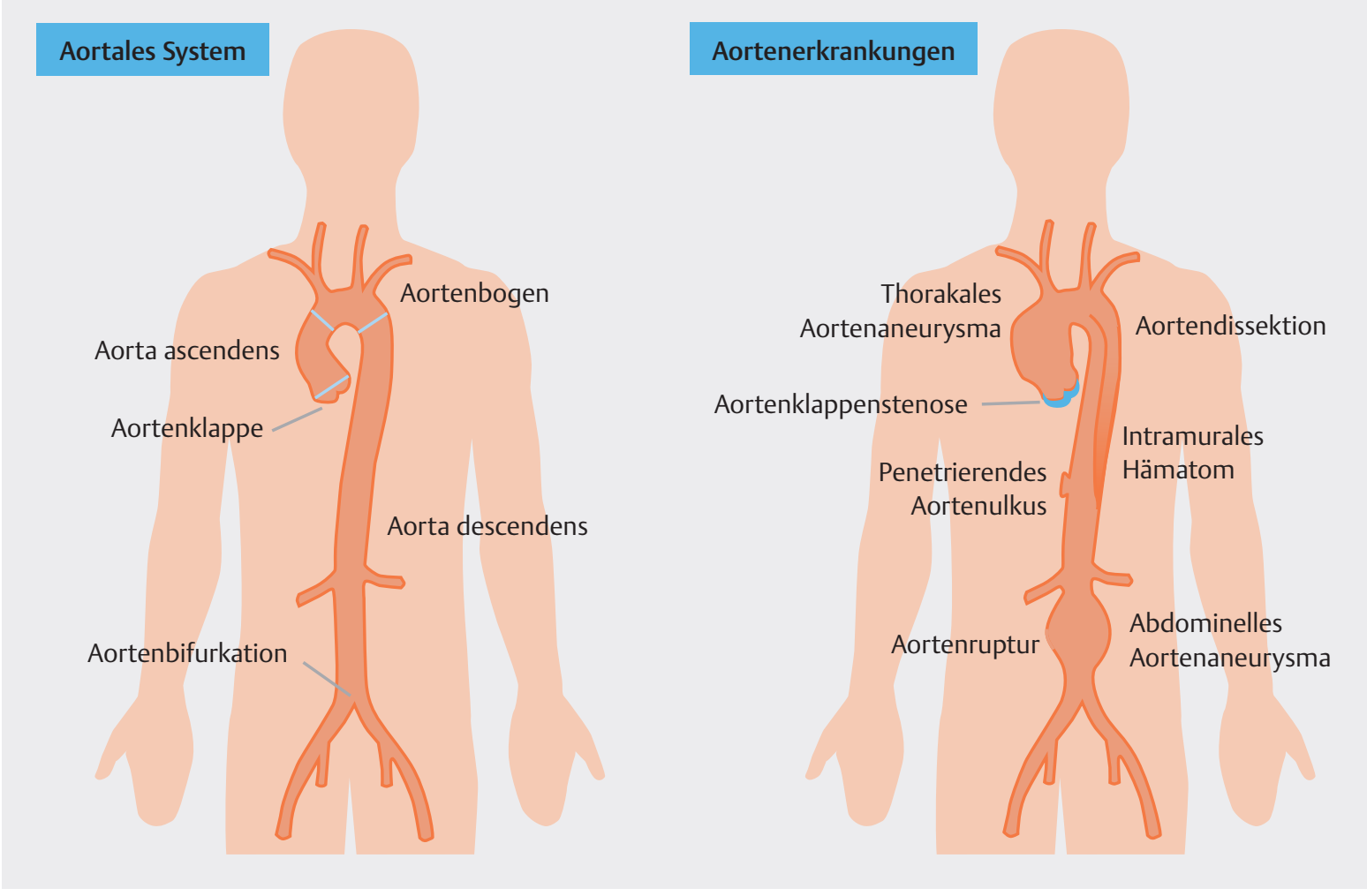

- Abb. 1 Aortales System und Aortenerkrankungen. Das aortale System verbindet das Herz mit der peripheren Zirkulation und besteht aus Aortenklappe, Aorta ascendens, Aortenbogen und Aorta descendens. Dargestellt sind Aortenerkrankungen wie die Aortenklappenstenose, das thorakale und abdominale Aortenaneurysma, Aortendissektion und -ruptur, intramurales Hämatom und penetrierendes Aortenulkus.

densein einer kontraktilen Reserve in der Stress-Echokardiografie vor TAVI ist bezüglich des klinischen Ergebnisses und der nachfolgenden Verbesserung der Ejektionsfraktion nicht zwingend erforderlich (siehe z. B. TOPAS-TAVIRegistry [1]).

Bezüglich des konventionellen Aortenklappenersatzes bei asymptomatischen Patienten mit normaler Ejektionsfraktion (EF) und ohne Blutdruckabfall unter Belastung waren bisher erhöhte Spiegel natriuretischer Peptide, ein Anstieg des mittleren Druckgradienten unter Belastung um $>20 \mathrm{mmHg}$ und eine deutliche Hypertrophie ohne Vorhandensein einer Hypertension (IIb C) relevant für die Indikationsstellung. Dies wurde aktuell konkretisiert: Sind 3-fach erhöhte BNP-Spiegel oder eine pulmonale Hypertonie vorhanden, sollte dies eine Entscheidung zur Therapie darstellen (Ila C). Die Versorgung von asymptomatischen Patienten mit einer höchstgradigen oder schnell progredienten Aortenklappenstenose $\left(V_{\max }>5,5 \mathrm{~m} / \mathrm{s}, V_{\max }-\right.$ Progression von $>0,3 \mathrm{~m} / \mathrm{s}$ pro Jahr $)$ bleibt eine Ila-C-Indikation. Interventionelle Valve-invalve-Prozeduren sollten abhängig vom operativen Risiko der Re-Operation, des Typs und der Größe der Prothese als Entscheidung in Herzteams getroffen werden (Empfehlung Ila C) [2].

\section{Ausdehnung der Indikation für die interven- tionelle Versorgung}

Wie die Therapievergleichsstudie PARTNER 2A zeigt, ist bei Patienten mit hochgradiger, symptomatischer Aortenklappenstenose mit intermediärem OP-Risiko der TAVR im Vergleich zum konventionellen Aortenklappenersatz (AKE) bezüglich des primären kombinierten Endpunktes nicht unterlegen [3]. Die LRT-Studie (LRT: Low Risk TAVR) befasst sich als eine der ersten mit dem Kollektiv mit niedrigem („low-risk“) perioperativem Risiko [4, 5]. Erste Datenanalysen der LRT-Studie [6] und des deutschen GARY-Registers [7] wie auch aus SURTAVI low risk $[8,9]$ und NOTION [10] legen nahe, dass TAVR bei Patienten mit hochgradiger Aortenklappenstenose und niedrigem Operationsrisiko im Vergleich zum chirurgischen Aortenklappenersatz ähnlich effektiv und sicher zu sein scheint.

Groß angelegte randomisierte Studien wie PARTNER 3, EVOLUT TAVR und DEDICATE werden weitere Daten zum Vergleich zwischen interventionellem und chirurgischem Aortenklappenersatz bei Niedrigrisiko-Patienten mit hochgradiger Aortenklappenstenose liefern. 


\section{Klinische Auswirkungen der aktuellen Optimierungen im Klappendesign}

Prothesentypen und -größen, die für TAVR eingesetzt werden, werden laufend weiterentwickelt. Neben dem Einsatz ballonexpandierender Prothesen stehen aktuell selbstexpandierende Prothesen im Fokus. Optimierungen im Klappendesign sollen unter anderem dabei helfen, die Komponente der postinterventionellen Aortenklappeninsuffizienz bei hoher Kalklast im Implantationsbereich zu minimieren. Ergebnisse der FORWARDStudie im 1-Jahres-Follow-up zeigen gute Sicherheit und Wirksamkeitsprofile der neuen Generation selbstexpandierender Aortenklappenprothesen bei geringer Mortalität und einer niedrigen Rate unerwünschter Ereignisse [11]. Dies spiegelt sich auch in der exzellenten hämodynamischen Performance und geringen Inzidenz paravalvulärer Leckagen neuerer Prothesen wider [12]. Gegenstand weiterer Verbesserungsansätze ist es, die Rate von postprozedural notwendigen Schrittmacherimplantationen zu minimieren. Ebenso soll das Verfahren weiter optimiert werden, indem die Zugangswege - insbesondere bei einem durch PAVK vaskulär vorerkrankten Patientenkollektiv - verkleinert werden. Aktuell sind kleinere Kaliber der Zugangswege bis zu einem Außendurchmesser (OD) von 12 French in Arbeit.

\section{Aortenklappeninsuffizienz}

Als neue IC-Empfehlung bei schwerer Aortenklappenregurgitation soll im interdisziplinären Herzteam bei ausgewählten Patienten eine Klappenrekonstruktion als Alternative zum Klappenersatz mit Reimplantation oder Remodeling mithilfe einer aortalen Anuloplastie diskutiert werden. Generell wird die operative Versorgung bei relevanter Aortenklappeninsuffizienz bei symptomatischen Patienten (Empfehlung IB) und bei asymptomatischen Patienten mit einer linksventrikulären EF $\leq 50 \%$ (IB) empfohlen, außerdem dann, wenn gleichzeitig eine aortokoronare Bypass-Operation, Operation der aszendierenden Aorta oder einer anderen Herzklappe (IC) geplant wird.

Kathetergestützt implantierte Klappenprothesen, die dediziert zur Behandlung der isolierten Aortenklappeninsuffizienz entwickelt werden, befinden sich für dieses Krankheitsbild in der klinischen Prüfung.

Klinische Relevanz

Sollte es evidenzbasiert zu einer Ausweitung der Indikation für TAVR auf ein Kollektiv mit niedrigem OPRisiko kommen, würde das den Einschluss einer großen zusätzlichen Patientengruppe bedeuten - unter der Voraussetzung, dass die Rate der postprozeduralen Schrittmacherpflicht niedrig ist und die Haltbarkeit der implantierten Klappen den Anforderungen gerecht wird [13].

\section{Erkrankungen der Aorta ascendens}

\section{Das akute Aortensyndrom}

In der aktuellen Leitlinie werden nun Erkrankungen der gesamten Aorta thematisiert, womit dem neu etablierten Konzept Rechnung getragen wird. Der Fokus liegt dabei auf der v. a. durch CT-Bildgebung standardisierten Bestimmung der Aortenpathologie. Integriert sind zudem Verfahrensanweisungen zur Behandlung des akuten Aortensyndroms [14, 15].

Unter einem akuten Aortensyndrom werden die traumatische Aortenverletzung, klassische sowie iatrogene Aortendissektion, die Aortenruptur, das intramurale Hämatom und penetrierendes Aortenulkus subsumiert. Handelt es sich dabei um stabile Patienten, dann helfen folgende Parameter dabei, die Wahrscheinlichkeit für das Vorliegen eines akuten Aortensyndroms einzuschätzen [15]:

- Hochrisikofaktoren (z. B. Marfan-Syndrom, Aortenklappenerkrankung, bekanntes Aneurysma),

- Hochrisikosymptome wie Brust- oder Rückenschmerzen und

- Untersuchungsbefunde (z. B. peripheres Perfusionsdefizit, Aortenklappeninsuffizienz).

\section{Aktuelles zur Bildgebung}

EKG-getriggerte CT-Aufnahmen erreichen heute für die Diagnose der Aortendissektion und des intramuralen Hämatoms eine Sensitivität von $100 \%$ und eine Spezifität von $98 \%$ [14]. Das MRT wird zum Teil auch als Screeningmethode eingesetzt, ist allerdings durch die eingeschränkte Darstellung von Gefäßverkalkungen und Stentstreben und die begrenzte Einsetzbarkeit bei Schrittmacherträgern limitiert. Dabei können der intimale flap bei Dissektionen und Blutflussturbulenzen in Entry/re-entry-Bereichen detektiert werden. Die transthorakale und transösophageale Echokardiografie stellen die Methoden der Wahl für die intraoperative Diagnostik, aber auch für den Einsatz auf der Intensivstation und im Schockraum dar.

\section{Erkrankungen der Aorta descendens}

\section{Aktuelles in Diagnostik und Therapie}

Die abdominelle Ultraschalluntersuchung ist weiterhin eine der Standardmodalitäten in der Bildgebung abdomineller Aortenerkrankungen. Bei Männern (Klasse IA) und Raucherinnen über 65 Jahren (Empfehlung Klasse IIb C) soll ein Screening auf ein abdominelles Aortenaneurysma mittels Sonografie durchgeführt werden $[15,16]$. Eine Ila-B-Empfehlung besteht dafür, in diesem Kollektiv bei jeder echokardiografischen Untersuchung auch die Bauchaorta zu untersuchen, was mittlerweile eine von 
der Krankenkasse akzeptierte Empfehlung darstellt, die vergütet wird. Dabei gilt:

- Bei einem Durchmesser < 45 mm ist eine jährliche Verlaufsbeobachtung ausreichend,

- bei einem Durchmesser von 45-55 mm und klinisch stabiler Situation wird eine halbjährliche Kontrolle empfohlen (Klasse IC) und

- bei einem symptomatischen abdominellen Aortenaneurysma ist die Therapie mittels EVAR (endovaskuläre Aortenreparatur) oder eine offene Operation indiziert (IA).

Bei der Therapie der Aorta descendens erhält die endovaskuläre Therapie eine höhere Bedeutung und soll gegenüber der operativen Versorgung favorisiert werden (Klasse lla C).

\section{Bildgebendes Follow-up: was, wann und für wen?}

Neben dem Screening ist auch das Follow-up insbesondere nach endovaskulären Eingriffen im ambulanten Sektor ein relevanter Baustein der Versorgung. Hierbei gilt es mögliche Folgen nach endovaskulärem Repair zu erkennen, wie beispielsweise die Entwicklung eines Endoleaks, Endograft-Migration oder Stentbruch. Das Vorhandensein eines Endoleaks, insuffiziente Abdichtung bei der ersten postoperativen Untersuchung und die ausbleibende Schrumpfung nach 1 Jahr sind starke Risikoprädiktoren für spät auftretende Komplikationen nach endovaskulärem Repair, was engmaschige Bildgebung nötig macht [17].

Nach offener chirurgischer Operation eines abdominellen Aortenaneurysmas ist eine gelegentliche Verlaufsbeobachtung mittels Ultraschall oder CT alle 5 Jahre ausreichend (Klasse IC); nach EVAR sollte nach 1 Monat, 6 und 12 Monaten sowie jährlich untersucht werden [15].

\section{Klinische Relevanz}

Von entscheidender klinischer Relevanz ist es, Screeninguntersuchungen durchzuführen und Hochrisikopatienten zu erkennen, bei denen eine frühzeitige Sanierung bei Aortenerkrankungen notwendig ist. Endovaskuläre Therapieansätze - ergänzt um individualisierte hybride Therapieoptionen - setzen ein bedarfsgerechtes Follow-up voraus.

\section{Interessenkonflikte}

Dr. med. Christine Quast: Research Funding: Forschungskommission Düsseldorf.

PD Dr. med. Sebastian Zimmer: Honoraria: Abbot, Abiomed, Astra Zeneca, Bayer, Böhringer Ingelheim, Medtronic, Novartis; Research Funding: DFG, BONFOR.
Dr. med. Matti Adam: Honoraria: Abbott, Bayer, Medtronic; Clinical Trials: Boston Scientific, Edwards, Medtronic; Research funding: DFG.

Prof. Dr. med. Stephan Baldus: Honoraria: Abbott, Astra, Bayer, Berlin Chemie, Cardiovalve, Daiichi Sankyo, Medtronic, Pfizer, Servier; Clinical Trials: Abbott, Astra, Boston Scientific, Cardiovalve, Edwards, Medtronic, Novartis, Pfizer, Servier; Research funding: DFG, Abbott, Actelion, Astra Zeneca, Bayer, Edwards, Pfizer.

Prof. Dr. med. Georg Nickenig: Honoraria for lectures or advisory boards: Abbott, AGA, AstraZeneca, Bayer, Berlin Cardiovalve, Chemie, Biosensus, Biotronic, BMS, Boehringer Ingelheim, Daiichi Sankyo, Edwards, Medtronic, Novartis, Pfizer, Sanofi Aventis, St. Jude; Participation in clinical trials: Abbott, AGA, AstraZeneca, Bayer, Berlin Chemie, Biosensus, Biotronic, BMS, Boehringer Ingelheim, Cardiovalve, Daiichi Sankyo, Edwards, Medtronic, Novartis, Pfizer, Sanofi Aventis, St. Jude; Research funding: DFG, BMBF, EU, Abbott, AGA, AstraZeneca, Bayer, Berlin Chemie, Biosensus, Biotronic, BMS, Boehringer Ingelheim, Daiichi Sankyo, Edwards, Medtronic, Novartis, Pfizer, Sanofi Aventis, St. Jude.

Prof. Dr. med. Malte Kelm: Honoraria: Bayer, Research funding: DFG, Philipps, Med. in NRW, Mars inc., Profil

\section{Autorinnen/Autoren}

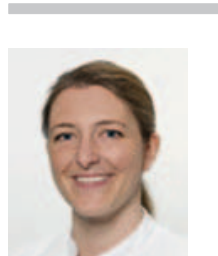

\section{Dr. med. Christine Quast}

ist Funktionsoberärztin in der Klinik für Kardiologie, Pneumologie und Angiologie des Universitätsklinikums Düsseldorf, Heinrich-HeineUniversität Düsseldorf.

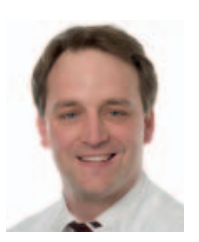

\section{Priv.-Doz. Dr. med. Sebastian Zimmer}

ist Oberarzt am Herzzentrum Bonn, Medizinische Klinik und Poliklinik II, Universitätsklinikum Bonn, Schwerpunkt Interventionelle Kardiologie, Notfall- und Intensivmedizin.

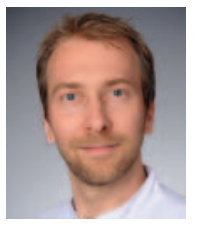

\section{Dr. med. Matti Adam}

ist Oberarzt der Klinik III für Innere Medizin Allgemeine und interventionelle Kardiologie, Elektrophysiologie, Angiologie, Pneumologie und internistische Intensivmedizin, Uniklinik Köln.

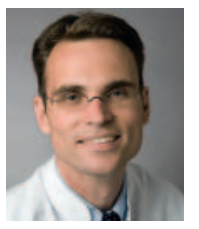

Univ.-Prof. Dr. med. Stephan Baldus

ist Direktor der Klinik III für Innere Medizin Allgemeine und interventionelle Kardiologie, Elektrophysiologie, Angiologie, Pneumologie und internistische Intensivmedizin, Uniklinik Köln.

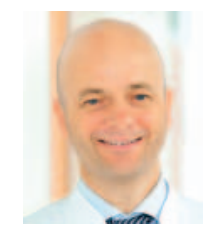

Univ.-Prof. Dr. med. Georg Nickenig

ist Direktor der Medizinischen Klinik und Poliklinik II des Herzzentrums Bonn, Universitätsklinik Bonn. 


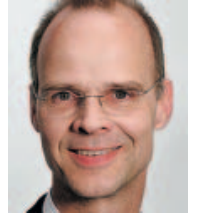

\section{Univ.-Prof. Dr. med. Malte Kelm}

ist Direktor der Klinik für Kardiologie, Pneumologie und Angiologie des Universitätsklinikums Düsseldorf, Heinrich-Heine-Universität Düsseldorf; Cardiovascular Research Institute Düsseldorf (CARID).

\section{Korrespondenzadresse}

\section{Dr. med. Christine Quast}

Universitätsklinikum Düsseldorf

Klinik für Kardiologie, Pneumologie und Angiologie

Moorenstr. 5

40225 Düsseldorf

Christine.Quast@med.uni-duesseldorf.de

\section{Literatur}

[1] Ribeiro HB, Lerakis S, Gilard M et al. Transcatheter Aortic Valve Replacement in Patients With Low-Flow, Low-Gradient Aortic Stenosis: The TOPAS-TAVI Registry. J Am Coll Cardiol 2018; 71 : $1297-1308$

[2] Baumgartner H, Falk V, Bax JJ et al. 2017 ESC/EACTS Guidelines for the management of valvular heart disease. European Heart Journal 2017; 38: 2739-2791

[3] Leon MB, Smith CR, Mack M] et al. Transcatheter or surgical aortic-valve replacement in intermediate-risk patients. N Engl J Med 2016; 374: 1609-1620

[4] Rogers T, Torguson R, Bastian R et al. Feasibility of transcatheter aortic valve replacement in low-risk patients with symptomatic severe aortic stenosis: Rationale and design of the Low Risk TAVR (LRT) study. Am Heart J 2017; 189: $103-109$

[5] Barker CM, Reardon M]. Should TAVR Replace Surgery for Aortic Stenosis in Low- to Intermediate-Risk Patients? Can J Cardiol 2017; 33: $1124-1128$

[6] Waksman R, Rogers T, Torguson R et al. Transcatheter Aortic Valve Replacement in Low-Risk Patients With Symptomatic Severe Aortic Stenosis. J Am Coll Cardiol 2018; 72: 2095-2105

[7] Bekeredjian R, Szabo G, Balaban Ü et al. Patients at low surgical risk as defined by the Society of Thoracic Surgeons Score undergoing isolated interventional or surgical aortic valve implantation: in-hospital data and 1-year results from the German Aortic Valve Registry (GARY). Eur Heart J 2018. doi:10.1093/eurheartj/ehy699
[8] Serruys PW, Modolo R, Reardon M et al. One-year outcomes of patients with severe aortic stenosis and an STS PROM of less than three percent in the SURTAVI trial. Eurolntervention 2018; 14: $877-883$

[9] Bagur R. Transcatheter aortic valve looking for low-risk patients: a post hoc analysis of SURTAVI keeps opening the door. Eurolntervention 2018; 14: 846-848

[10] Søndergaard L, Steinbrüchel DA, Ihlemann N et al. Two-Year Outcomes in Patients With Severe Aortic Valve Stenosis Randomized to Transcatheter Versus Surgical Aortic Valve Replacement: The All-Comers Nordic Aortic Valve Intervention Randomized Clinical Trial. Circ Cardiovasc Interv 2016; 9: e003665

[11] Manoharan G, Van Mieghem NM, Windecker S et al. 1-Year Outcomes With the Evolut R Self-Expanding Transcatheter Aortic Valve: From the International FORWARD Study. JACC Cardiovasc Interv 2018; 11: 2326 - 2334

[12] Hellhammer K, Piayda K, Afzal S et al. The Latest Evolution of the Medtronic CoreValve System in the Era of Transcatheter Aortic Valve Replacement: Matched Comparison of the Evolut PRO and Evolut R. JACC Cardiovasc Interv 2018; 11: 2314 2322

[13] Jilaihawi H, Williams M. Surgical Versus First-Generation SelfExpanding Transcatheter Aortic Valve Replacement: Is TAVR More Durable? J Am Coll Cardiol 2018; 72: 2697-2700

[14] Erbel R, Aboyans V, Boileau C et al. 2014 ESC Guidelines on the diagnosis and treatment of aortic diseases: Document covering acute and chronic aortic diseases of the thoracic and abdominal aorta of the adult. The Task Force for the Diagnosis and Treatment of Aortic Diseases of the European Society of Cardiology (ESC). Eur Heart ] 2014; 35: 2873-2926

[15] Erbel R, Eggebrecht H, Falk V et al. Kommentar zur 2014-Leitlinie der Europäischen Gesellschaft für Kardiologie (ESC) zur Diagnose und Therapie von Aortenerkrankungen. Kardiologe 2015; 9: 348-353

[16] Takagi H, Goto SN, Matsui M et al. A further meta-analysis of population based screening for abdominal aortic aneurysm. J Vasc Surg 2010; 52: 1103-1108

[17] Goncalves FB. Post-EVAR Surveillance Considerations, Evaluating and stratifying risks based on currently available data. Endovascular today 2017; 16: 90-92 